\title{
The RBI Mutation Spectrum and Genetic Management Consultation in Pediatric Patients with Retinoblastoma in Beijing, China
}

\author{
Ying $X_{i e}^{1,2}$ \\ Xiao-Lin Xu' \\ Wen-Bin Wei \\ 'Beijing Tongren Eye Center, Beijing Key \\ Laboratory of Intraocular Tumor \\ Diagnosis and Treatment, Beijing \\ Ophthalmology and Visual Sciences Key \\ Lab, Medical Artificial Intelligence \\ Research and Verification Key Laboratory \\ of the Ministry of Industry and \\ Information Technology, Beijing Tongren \\ Hospital, Capital Medical University, \\ Beijing, 100730, People's Republic of \\ China; ${ }^{2}$ Department of Opthalmology, \\ Shanxi Provincial People's Hospital, \\ Taiyuan, 0300 I2, People's Republic of \\ China
}

Correspondence: Wen-Bin Wei Beijing Tongren Eye Center, Beijing Key Laboratory of Intraocular Tumor Diagnosis and Treatment, Beijing Ophthalmology and Visual Sciences Key Lab, Medical Artificial Intelligence Research and Verification Key Laboratory of the Ministry of Industry and Information Technology, Beijing Tongren Hospital, Capital Medical University, No. I of Dong Jiao Min Lane, Dongcheng District, Beijing, I00730, People's

Republic of China

Tel +86 I0 58269516

Fax +86 1065125617

Email weiwbl023@163.com
Objective: The present study screened the structural mutations of the retinoblastoma (RB1) gene using gene capture and a preliminary exploration of the correlation between the genotypes and phenotypes.

Methods: A total of 45 formalin-fixed paraffin-embedded (FFPE) tissue samples and 12 peripheral venous blood samples from patients with retinoblastoma (RB) confirmed by pathological examination at Beijing Tongren Hospital were collected between May 2019 and May 2021. DNA from the samples was extracted, sequenced, and analyzed to detect the mutations in the RB1 gene by designing the targeted capture probes for exons and the flanking sequences of the gene.

Results: Of the 45 FFPE tissue samples, 23 were from male patients and 22 were from female patients, all aged between 4 months and 10 years, with an average age of $2.5 \pm 1.3$ years. Two of these patients had bilateral RB and 43 had unilateral RB (23 in the right eye and 20 in the left eye). Of the 12 peripheral venous blood samples, 7 were from male patients and 5 were from female patients, all aged between 8 months and 4 years, with an average age of $1.3 \pm 0.9$ years. Two of these patients had bilateral RB and 10 had unilateral RB ( 8 in the right eye and 2 in the left eye). Three de novo pathogenic mutations were found in the FFPE tissues, along with one de novo potentially pathogenic mutation, while three de novo potentially pathogenic mutations were found in the blood samples.

Conclusion: Gene capture is a low-cost and efficient method for the gene sequencing of RB. A total of seven de novo mutations were identified through mutation testing of the pathogenic gene RB1 in 56 pediatric patients with RB. This complemented the mutation spectrum of the RB1 gene and helped to improve the molecular diagnosis of RB, thereby providing a basis for genetic counseling and prediction of the clinical phenotype, as well as for the genetic testing of the offspring of patients with RB.

Clinical Registration Number: ChiCTR-EPC-17013892.

Keywords: retinoblastoma, RB1 gene, gene capture

\section{Introduction}

Retinoblastoma (RB) is the most common primary intraocular malignancy in infants and children, accounting for $3 \%$ of all malignant tumors in pediatric patients, and the second most common malignant tumor in pediatrics overall. ${ }^{1,2}$ The global incidence of RB is 1 in 15,000-20,000 live births, ${ }^{3}$ and the incidence in China is 1 in 23,160. ${ }^{4} \mathrm{RB}$ was first reported by the Dutch internist Pawius in 1597, and the first case in China was reported by Huade Bi in 1921. 5,6 The increase in the incidence of $\mathrm{RB}$ is due to improved diagnosis and a gradual increase in recognition 
and awareness, which have greatly increased the detection rate, and the prevalence of early diagnosis and the rational treatment of patients, which have increased the cure rate. Due to the increased cure rate, there is also now a higher chance of survivors passing on the pathological gene to their offspring.

There are two types of RB - hereditary and non-hereditary - with hereditary RB accounting for approximately $45 \%$ of cases and non-hereditary RB accounting for approximately $55 \%{ }^{7}$ Hereditary RB is caused by germcell mutation and manifests as bilateral RB (accounting for $80 \%$ of cases) or unilateral multiple RB (accounting for $15 \%$ of cases), of which approximately $5 \%$ can be accompanied by intracranial tumors, called trilateral RB. ${ }^{8}$ Most patients with hereditary RB develop a second malignant neoplasm several years later. ${ }^{9}$ Non-hereditary RB is caused by somatic mutations and mostly manifests as unilateral RB.

Although RB is a serious risk to the life of patients, the survival rate in developed countries can be as high as $95 \%$, and more than $90 \%$ of pediatric patients with RB retain visual function in at least one eye. ${ }^{10}$ However, for many pediatric patients in China, the tumor is already large by the time RB is diagnosed, making it difficult - and even life threatening - to preserve the eye.

The RB1 gene was the first tumor-suppressor gene to be discovered in humans. Its discovery is recognized as an important milestone in the study of human oncology and the cell cycle. The gene is located at $13 \mathrm{q} 14$, with a total length of approximately $180 \mathrm{~kb}$ and 27 exons, encoding an RB protein (pRB) with 928 amino acid residues. ${ }^{11}$ The structure of $\mathrm{pRB}$ is divided into three domains, composed of an N-terminus, an $\mathrm{R}$ motif, an $\mathrm{A} / \mathrm{B}$ pocket, and a C-terminus. ${ }^{12}$ Mutations in the RB1 gene deactivate the $\mathrm{pRB}$, resulting in abnormal cell-cycle division and the transformation of benign retinal tumors into malignant RB. $^{13}$

Current genetic testing methods involve extracting high-quality DNA from the blood and/or tumor tissue and using quantitative polymerase chain reaction (PCR), gene chips, or high-throughput sequencing to detect the presence of variants, such as rearrangements, deletions, and insertions in the genes; allele-specific PCR is also used for the known common gene-mutated loci, along with the sequencing of all exons and the intron shear site of the RB1 gene. Based on current understanding and technology, $5 \%$ of patients with $\mathrm{RB}$ are not found to have pathogenic gene mutations. ${ }^{14}$
In the present study, a new-generation sequencing platform based on sequence capture of the target region was used to sequence the RB1 gene in DNA extracted from the formalin-fixed paraffin-embedded (FFPE) tissues and peripheral venous blood of pediatric patients with RB. This platform can detect both the copy number and single-base mutations and has the advantages of high sensitivity, high specificity, high throughput, low costs, and rapid results. The findings from this statistical analysis of the RB1 gene contribute to current understanding of the high-frequency mutated loci of the RB1 gene in patients with RB in China, which will help with the early diagnosis of RB in pediatric patients and guide genetic consultation for families with RB.

\section{Subjects and Methods Study Subjects}

DNA samples from the FFPE tissues of 45 pediatric patients with RB and from the peripheral venous blood of 12 pediatric patients with RB were studied between May 2019 and May 2021. One patient underwent simultaneous blood sample and FFPE tissue testing, so there were 27 female patients and 29 male patients enrolled in total.

The FFPE tissues were histopathologically diagnosed, and the clinical diagnosis of RB was made by clinical examination, radiological examination (ultrasound/computed tomography/magnetic resonance imaging), and RetCam imaging.

\section{Methods}

\section{Collection of Blood Samples and Concentration Detection}

A total of $2 \mathrm{~mL}$ of venous blood was collected from each patient, placed in a test tube, and anticoagulated with ethylenediaminetetraacetic acid. The DNA was extracted using a FlexiGene DNA Kit (Qiagen, Germany) and stored at $-80^{\circ} \mathrm{C}$ for further detection. DNA content was determined using a NanoDrop 2000 ultra-micro spectrophotometer (Thermo Fisher Scientific, USA).

\section{Collection of FFPE Tissues and Concentration Detection}

Excess paraffin on each FFPE tissue was removed with a scalpel, and the sample was sliced to a thickness of 10 $\mu \mathrm{m}$; the first 2-3 outside-exposed and air-exposed slices were discarded, and 1-2 internal slices were maintained for DNA extraction. The slices were immediately placed in $2 \mathrm{~mL}$ EP centrifuge tubes, and DNA was extracted using 
a FlexiGene DNA Kit (Qiagen, Germany) and stored at $80^{\circ} \mathrm{C}$ for further detection. DNA content was determined using a NanoDrop 2000 ultra-micro spectrophotometer (Thermo Fisher Scientific, USA).

\section{Probe Design and Target-Gene Sequencing}

Using the Online Mendelian Inheritance in Man database (OMIM), Human Gene Mutation Database (HGMD), and Leiden Open Variation Database 3.0 (LOVD) as references, target-capture probes targeting the exons and flanking sequences ( $\pm 10 \mathrm{bp}$ ) of the RB1 gene were designed using the SureDesign online design tool (Agilent, USA). The target-gene capture libraries were prepared using an Agilent SureSelect Target Enrichment System Kit (Agilent, USA) on the DNA samples from the 56 patients, after which the samples from the sequencing libraries were tested using an Agilent 2100 Bioanalyzer with DNA chips (Agilent, USA) and DNA 1000 Reagent (Agilent, USA). A NextSeq 500 sequencer (Illumina, USA) was then used to sequence the DNA samples according to the concentration and sequencing depth requirements of the captured library.

\section{Data Analysis and Evaluation}

The raw-data FASTQ files generated by the sequencing platform were analyzed using RTA software (Illumina, USA), CASAVA software v. 1.8.2 (Illumina, USA), and the genetic analysis toolkit bioinformatics software to assess the sequencing quality. The evaluation parameters included total number of reads, proportion of matches to human genome sequences, proportion located within the target sequence of the target gene, average sequencing depth, proportion of sequenced regions greater than $20 \times$ depth, and coverage uniformity analysis. After generating single-nucleotide variant (SNV) reports, variant annotation was performed using PolyPhen 2.2.2 software, Annotate Variation software, the HGMD database, the LOVD database, the Single Nucleotide Polymorphism Database (dbSNP), and the 1000 Genomes Project database.

\section{Results}

\section{General Characteristics}

The general characteristics of the 56 patients are shown in Tables 1 and 2. Most of the probands were classified as group D or E (according to the International Classification of Intraocular Retinoblastoma) at the time of diagnosis, accounting for $96 \%$, with two cases in the FFPE group being classified as group $\mathrm{C}$, accounting for $4 \%$.
Table I General Clinical Profiles of RB Probands in This Study (FFPE)

\begin{tabular}{|c|c|c|c|}
\hline Characteristics & $\begin{array}{c}\text { Positive } \\
(n=40)\end{array}$ & $\begin{array}{c}\text { Negative } \\
(n=1)\end{array}$ & $\begin{array}{c}\text { Else } \\
(n=4)\end{array}$ \\
\hline Age $(y)$ & $2.5 \pm 1.3$ & & \\
\hline \multicolumn{4}{|l|}{$\operatorname{Sex}(n)$} \\
\hline Male (\%) & $52.5 \%$ & & $50 \%$ \\
\hline Female (\%)) & $47.5 \%$ & $100 \%$ & $50 \%$ \\
\hline \multicolumn{4}{|l|}{ Laterality (n) } \\
\hline Unilateral (\%) & $95 \%$ & $100 \%$ & $100 \%$ \\
\hline Right eye & 22 & & I \\
\hline Left eye & 16 & I & 3 \\
\hline Bilateral (\%) & $5 \%$ & 0 & 0 \\
\hline \multicolumn{4}{|l|}{ IIRC Stage (n) } \\
\hline A & & & \\
\hline B & & & \\
\hline C & 2 & & \\
\hline $\mathrm{D}$ & 16 & 1 & I \\
\hline$E$ & 22 & & 3 \\
\hline
\end{tabular}

Table 2 General Clinical Profiles of RB Probands in This Study (Blood)

\begin{tabular}{|l|c|c|c|}
\hline Characteristics & $\begin{array}{c}\text { Positive } \\
(\mathbf{n}=1 \mathbf{2})\end{array}$ & $\begin{array}{c}\text { Negative } \\
(\mathbf{n}=\mathbf{0})\end{array}$ & $\begin{array}{c}\text { Else } \\
(\mathbf{n}=\mathbf{0})\end{array}$ \\
\hline Age (y) & $1.3 \pm 0.9$ & & \\
\hline Sex (n) & & & \\
Male (\%) & $58.3 \%$ & & \\
Female (\%)) & $41.7 \%$ & & \\
\hline Laterality (n) & & & \\
Unilateral (\%) & $83.3 \%$ & & \\
Right eye & 8 & & \\
Left eye & 2 & & \\
Bilateral (\%) & $16.7 \%$ & & \\
\hline IIRC Stage (n) & & & \\
A & & & \\
B & & & \\
C & & & \\
D & & & \\
E & & & \\
\hline
\end{tabular}

Results of the Gene Detection in the FFPE Tissue Samples and Blood Samples FFPE Tissue Samples

One case in the FFPE tissue samples had negative results, four cases were found to be non-RB1 mutations, and the remaining 40 cases showed $\mathrm{RB} 1$ mutations, with a positivity of $88.9 \%$. The 44 cases are summarized in Table 3 . 


\begin{tabular}{|c|c|c|c|c|c|c|c|c|c|c|c|c|c|c|c|c|c|c|c|c|c|c|}
\hline  & I & $\Xi$ & I & $\Xi$ & $I$ & $\Xi$ &  & $\Xi$ & I & $\Xi$ & 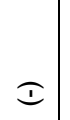 & I & I & $\Xi$ & $\Xi$ & $\Xi$ & $\Xi$ & $\Xi$ & $\Xi$ & $\Xi$ & $\mathbb{I}$ & II \\
\hline 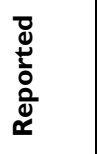 & $\stackrel{n}{\lambda} \stackrel{0}{\lambda}$ & $=$ & $=\lambda$ & $\stackrel{\infty}{\lambda}$ & $\stackrel{n}{\lambda}$ & $\stackrel{\sigma}{\lambda}$ & $\AA_{\lambda}$ & $\bar{\gamma}_{\lambda}$ & ${ }^{n} x_{1}$ & $\stackrel{n}{\lambda}$ & $=$ & $\tilde{\lambda}_{\lambda}$ & $\nexists_{\lambda}$ & $\stackrel{ \pm}{\lambda}$ & $\stackrel{n}{\lambda}$ & $\pi_{\lambda}$ & $\stackrel{2}{x}$ & $\stackrel{\overbrace{\lambda}}{\lambda}$ & $\vec{\lambda}_{\lambda}$ & $\stackrel{\lambda}{\lambda}$ & $=$ & ${ }_{\lambda}^{\lambda} \stackrel{H}{\lambda}_{\lambda}$ \\
\hline 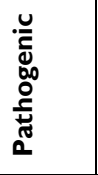 & $a \quad a$ & ב & a & a & 9 & $a$ & a & $a$ & $a$ & 9 & a & $a$ & $a$ & $a$ & $a$ & $a$ & a & $a$ & $a$ & $a$ & $a$ & $a$ \\
\hline 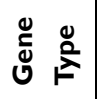 & $\stackrel{\ddot{\Xi}}{\ddot{\Xi}}$ & $\begin{array}{l}\varepsilon \\
\stackrel{\varepsilon}{\Phi}\end{array}$ & $\begin{array}{l}\varepsilon \\
\text { ह } \\
\underline{\Phi}\end{array}$ & $\underset{\underline{\mathbf{v}}}{\underline{\underline{n}}}$ & $\stackrel{\ddot{\Xi}}{\Phi}$ & $\begin{array}{l}\varepsilon \\
\stackrel{\varepsilon}{\Phi}\end{array}$ & 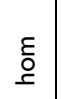 & $\begin{array}{l}\varepsilon \\
\stackrel{\varepsilon}{0} \\
\end{array}$ & $\begin{array}{l}\varepsilon \\
\text { g }\end{array}$ & $\begin{array}{l}\varepsilon \\
\text { g }\end{array}$ & $\stackrel{\breve{\Xi}}{\Phi}$ & है & $\stackrel{\ddot{\Xi}}{\Phi}$ & 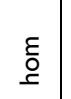 & $\begin{array}{l}\varepsilon \\
\stackrel{\varepsilon}{0}\end{array}$ & $\begin{array}{l}\varepsilon \\
\end{array}$ & $\underset{\underline{\mathbf{v}}}{\underline{4}}$ & $\stackrel{\stackrel{\Xi}{\Xi}}{\Phi}$ & $\begin{array}{l}\varepsilon \\
\stackrel{\varepsilon}{\prime}\end{array}$ & $\begin{array}{l}\varepsilon \\
\stackrel{\varepsilon}{\rho}\end{array}$ & $\begin{array}{l}\varepsilon \\
\stackrel{\varepsilon}{\prime}\end{array}$ & 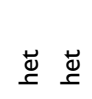 \\
\hline $\begin{array}{l}\tilde{\sigma} \\
\text { 㐫 } \\
\end{array}$ & $\simeq \approx$ & i & $\infty$ & $=$ & $\nabla$ & 0 & ㄱ. & $\underline{a}$ & $\infty$ & $\stackrel{m}{\sim}$ & $\underline{0}$ & $\ddot{\sim}$ & $\ddot{\sim}$ & \pm & $\ddot{\sim}$ & - & $\sigma$ & $\simeq$ & $\simeq$ & $\infty$ & $=$ & $\simeq$ i \\
\hline 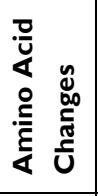 & 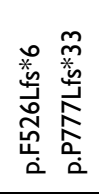 &  & $\begin{array}{l}\underset{x}{a} \\
\stackrel{x}{a} \\
\dot{\alpha}\end{array}$ & 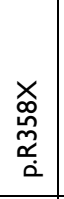 & 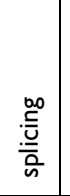 & 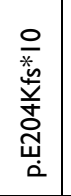 & $\begin{array}{l}\underset{x}{x} \\
\text { o. } \\
\text { on } \\
\dot{i}\end{array}$ & 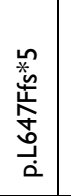 & $\begin{array}{l}\underset{x}{\mathfrak{X}} \\
\underset{\sim}{\stackrel{x}{a}} \\
\dot{a}\end{array}$ & 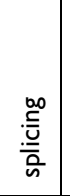 & 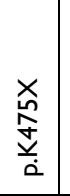 & $\begin{array}{l}\underset{x}{x} \\
\infty \\
\alpha \\
\alpha \\
\vdots\end{array}$ & $\begin{array}{l}\underset{n}{\tilde{N}} \\
\stackrel{\infty}{\circ} \\
\dot{a}\end{array}$ &  & 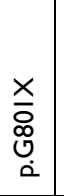 & 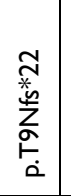 & 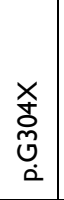 &  & $\begin{array}{l}\stackrel{n}{0} \\
\stackrel{\underline{\underline{u}}}{\bar{n}}\end{array}$ & 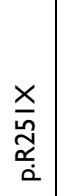 & 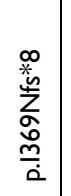 & 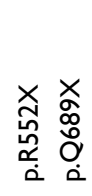 \\
\hline 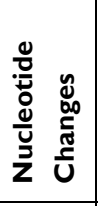 & 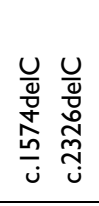 & 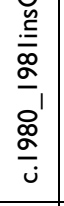 & $\begin{array}{r}\stackrel{n}{\hat{U}} \\
\stackrel{n}{\mathrm{u}} \\
\end{array}$ & 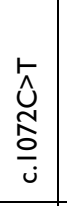 & 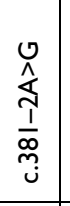 & 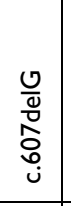 & 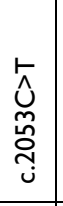 & 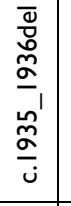 & $\frac{\mathfrak{r}}{\stackrel{\hat{U}}{\hat{N}}}$ & 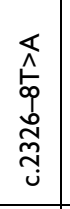 & 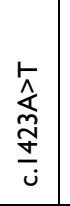 & 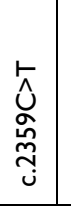 & 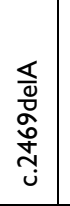 & 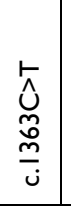 & 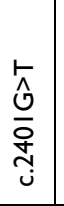 & 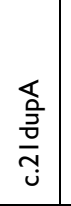 & $\begin{array}{l}\frac{\hat{\lambda}}{\hat{y}} \\
\frac{\partial}{\sigma} \\
\dot{c}\end{array}$ &  & $\begin{array}{l}\varangle \\
\hat{0} \\
\frac{\sigma}{1} \\
\sigma \\
\frac{\sigma}{\sigma} \\
\dot{v}\end{array}$ & $\frac{\stackrel{n}{\hat{U}}}{\stackrel{n}{\hat{v}}}$ & 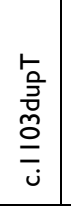 & 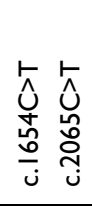 \\
\hline t̀ & $\underline{m}$ & $\underline{m}$ & $\underline{m}$ & $\underline{m}$ & $\underline{m}$ & $\underline{m}$ & $\underline{m}$ & $\underline{m}$ & $\underline{m}$ & $\underline{m}$ & $\underline{m}$ & $\underline{m}$ & $\underline{m}$ & $\underline{m}$ & $\underline{m}$ & $\underline{m}$ & $\underline{m}$ & $\underline{m}$ & $\underline{m}$ & $\underline{m}$ & $\underline{m}$ & $\underline{m} \underline{m}$ \\
\hline טّ̊ & $\overline{\underline{m}}$ & $\overline{\widetilde{q}}$ & $\overline{\underline{\infty}}$ & $\overline{\underline{\infty}}$ & $\overline{\underline{a}}$ & $\overline{\widetilde{\rho}}$ & $\bar{\alpha}$ & $\overline{\underline{\infty}}$ & $\overline{\underline{a}}$ & $\overline{\underline{\alpha}}$ & $\overline{\underline{m}}$ & $\overline{\underline{a}}$ & $\overline{\underline{m}}$ & $\overline{\underline{m}}$ & $\begin{array}{l}\bar{\infty} \\
\propto\end{array}$ & $\overline{\underline{m}}$ & $\overline{\widetilde{\infty}}$ & $\overline{\underline{\alpha}}$ & $\overline{\underline{\alpha}}$ & $\overline{\mathscr{\infty}}$ & $\overline{\underline{\alpha}}$ & 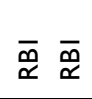 \\
\hline 离 & 0 & ш & 0 & س & 0 & ш & $u$ & 口 & 0 & 0 & ш & س & 0 & ш & ш & $u$ & 口 & ш & 0 & ш & ш & س \\
\hline 品 & $\lambda$ & $\hat{m}$ & $\vec{\lambda}$ & $\underline{\lambda}$ & ते & $\lambda$ & $\lambda$ & $\xi$ & ઢે & $\vec{f}$ & $\vec{\lambda}$ & $\vec{\lambda}$ & సे & $\lambda$ & ते & $\lambda$ & $\vec{m}$ & กे & ह & ते & $\lambda$ & $\lambda$ \\
\hline 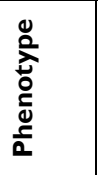 & $\supset$ & $\supset$ & $\supset$ & $\supset$ & $\supset$ & $\supset$ & $\supset$ & $\supset$ & $\supset$ & $\supset$ & $\supset$ & $\supset$ & $\supset$ & $\supset$ & $\supset$ & $\supset$ & $\supset$ & $\supset$ & $\supset$ & $\supset$ & $\supset$ & $\supset$ \\
\hline 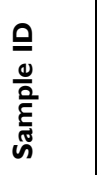 & 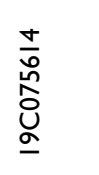 & $\begin{array}{l}\hat{0} \\
\hat{\circ} \\
\hat{0} \\
\alpha\end{array}$ & $\begin{array}{l}\frac{\infty}{0} \\
\hat{م} \\
\hat{0} \\
\alpha\end{array}$ & 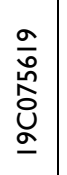 & 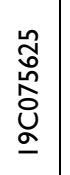 & 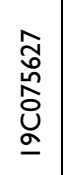 &  & $\begin{array}{l}\text { ָ̃ } \\
\bar{\alpha} \\
\text { ర్ } \\
\underline{-}\end{array}$ & $\begin{array}{l}\stackrel{\circ}{\circ} \\
\stackrel{+}{+} \\
\bar{\alpha} \\
\alpha\end{array}$ &  & 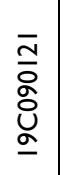 & 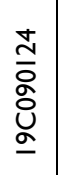 & 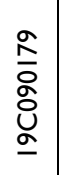 & 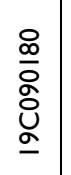 & $\begin{array}{l}\bar{\infty} \\
\bar{\alpha} \\
\bar{\delta} \\
\swarrow\end{array}$ & $\begin{array}{l}\stackrel{\infty}{\infty} \\
\frac{0}{2} \\
\stackrel{\delta}{0} \\
\alpha\end{array}$ & 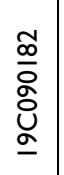 & $\begin{array}{l}\stackrel{\circ}{\circ} \\
\overline{0} \\
\text { ర్ } \\
\alpha\end{array}$ &  & 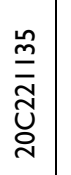 & 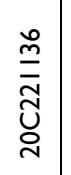 & 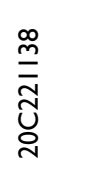 \\
\hline$\underline{z}$ & - & $\sim$ & $m$ & + & in & 0 & $n$ & $\infty$ & $\sigma$ & 으 & $=$ & $\simeq$ & $\underline{m}$ & \pm & $\underline{\underline{n}}$ & $\stackrel{\circ}{0}$ & $\simeq$ & $\underline{\infty}$ & $\underline{a}$ & ㅇ. & $\bar{\lambda}$ & $\pi$ \\
\hline
\end{tabular}




\begin{tabular}{|c|c|c|c|c|c|c|c|c|c|c|c|c|c|c|c|c|c|c|c|c|}
\hline$I$ & I & I & I & $I$ & $\mp$ & I & $I$ & $I$ & $I$ & I & I & I & I & I & I & I & $\Xi$ & $I I I$ & I & $I I I$ \\
\hline${ }_{\lambda}$ & $=$ & $\stackrel{\lambda}{\lambda}$ & $\overbrace{\lambda}$ & $\stackrel{n}{\lambda}$ & $\overbrace{\lambda}^{\infty}$ & $\overbrace{\lambda}$ & ${ }^{2} \lambda$ & ${ }_{\lambda}$ & $=>$ & ${ }_{\lambda}$ & ${ }_{\lambda}$ & $=$ & $=1$ & $=$ & $\stackrel{\infty}{\lambda}$ & $\mathrm{N}_{\lambda}^{ \pm}$ & $m_{\lambda}$ & $=c$ & $=$ & $=\varepsilon$ \\
\hline$a$ & 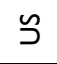 & a. & $a$ & $a$ & a & 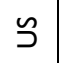 & $a$ & 0 & a & $a$ & $a$ & a & a & 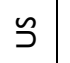 & a & 9 & a & 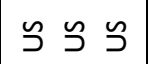 & $\mathscr{د}$ & 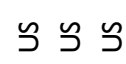 \\
\hline$\stackrel{\ddot{\Xi}}{\Phi}$ & $\stackrel{\ddot{\Xi}}{\Phi}$ & $\stackrel{\ddot{\Xi}}{\Phi}$ & $\stackrel{\ddot{\Xi}}{\Xi}$ & 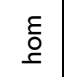 & $\stackrel{\ddot{\otimes}}{\Phi}$ & है & $\begin{array}{l}\varepsilon \\
\stackrel{g}{\rho}\end{array}$ & है & 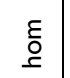 & $\begin{array}{l}\varepsilon \\
\stackrel{\rho}{\rho}\end{array}$ & 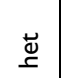 & $\stackrel{\breve{\Xi}}{\Xi}$ & है & 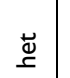 & Е & $\stackrel{\breve{\Xi}}{\check{\Phi}}$ &  & 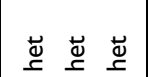 & 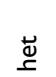 & 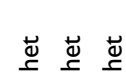 \\
\hline$\infty$ & - & $\infty$ & \pm & $\underline{\underline{n}}$ & $\infty$ & i & $\leq$ & 으 & $\simeq$ & $\underline{\infty}$ & 으 & $n$ & 으 & - & $\underline{\underline{n}}$ & i & 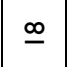 & $\wedge 00$ & $\circ$ & $=00$ \\
\hline 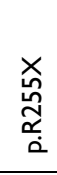 & $\frac{0}{d}$ & 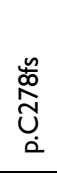 & $\begin{array}{l}\frac{00}{.0} \\
\frac{. \overline{0}}{\bar{y}} \\
\end{array}$ & 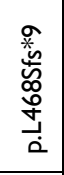 & 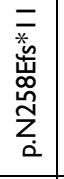 & 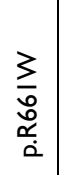 & 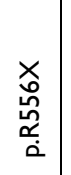 & $\left|\begin{array}{c}x \\
\stackrel{x}{0} \\
\underset{\alpha}{\alpha} \\
i\end{array}\right|$ &  & $\begin{array}{l}\underset{x}{\alpha} \\
\hat{\alpha} \\
\alpha \\
\vdots \\
\dot{\alpha}\end{array}$ & 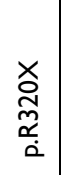 & 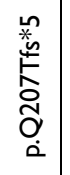 & 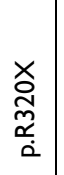 & 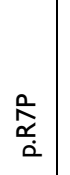 & 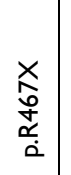 & 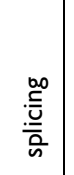 & 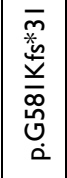 & 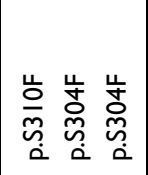 & 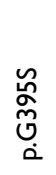 & 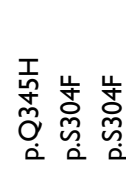 \\
\hline 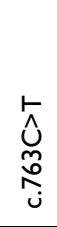 & 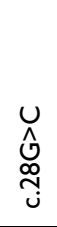 & 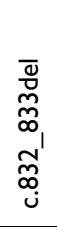 & 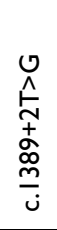 & 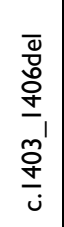 & 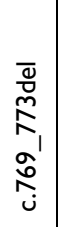 & $\begin{array}{l}\frac{\mathfrak{\hat { U }}}{o} \\
\frac{\alpha}{\dot{\sigma}}\end{array}$ &  & 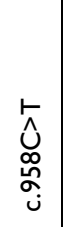 & 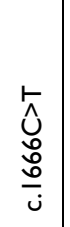 & 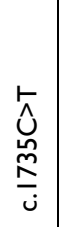 & 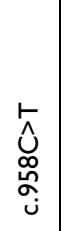 & 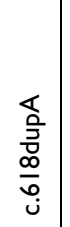 & $\begin{array}{l}\stackrel{h}{\hat{o}} \\
\text { og } \\
\text { un }\end{array}$ & 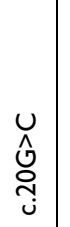 &  & $\begin{array}{l}\stackrel{\nwarrow}{\hat{U}} \\
\frac{1}{o} \\
\frac{\rho}{v}\end{array}$ & 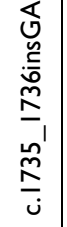 & 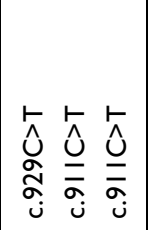 & 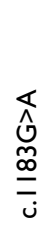 & 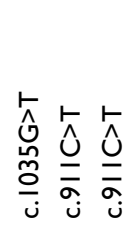 \\
\hline$\underline{m}$ & $\underline{m}$ & $\underline{m}$ & $\underline{m}$ & $\underline{m}$ & $\underline{m}$ & $\underline{m}$ & $\underline{m}$ & $\underline{m}$ & $\underline{m}$ & $\underline{m}$ & $\underline{m}$ & $\underline{m}$ & $\underline{m}$ & $\underline{m}$ & $\underline{m}$ & $\underline{m}$ & $\underline{m}$ & $\succ>>$ & $\simeq$ & $a>>$ \\
\hline$\overline{\mathscr{m}}$ & $\begin{array}{l}\bar{\alpha} \\
\propto\end{array}$ & $\overline{\underline{\infty}}$ & $\begin{array}{l}\bar{\alpha} \\
\propto\end{array}$ & $\overline{\underline{m}}$ & $\bar{\alpha}$ & 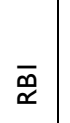 & 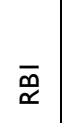 & $\overline{\underline{\alpha}}$ & $\overline{\underline{\alpha}}$ & 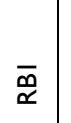 & $\overline{\underline{\alpha}}$ & $\overline{\underline{\infty}}$ & $\overline{\underline{\infty}}$ & $\bar{\Phi}$ & $\overline{\underline{\infty}}$ & $\overline{\underline{\infty}}$ & $\bar{\Phi}$ & 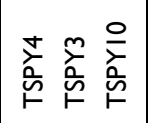 & 空 & 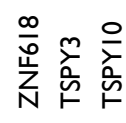 \\
\hline 0 & ш & ш & ш & 0 & w & w & $w$ & w & $w$ & ш & ш & 0 & w & 口 & 口 & 0 & 0 & w & ш & w \\
\hline$\hat{m}$ & $\underline{-}$ & $\lambda$ & $\lambda$ & ડે & $\lambda$ & $\lambda$ & oิ & $\underline{-}$ & 入 & $\lambda$ & $\underline{\lambda}$ & $\hat{m}$ & $\widehat{m}$ & $\underline{\lambda}$ & $\xi$ & $\geq$ & $\bar{m}$ &  & $\underline{\lambda}$ & $\lambda$ \\
\hline$\supset$ & $\supset$ & $\supset$ & $\supset$ & $\supset$ & $\infty$ & $\supset$ & $\supset$ & $\supset$ & $\supset$ & د & $\supset$ & $\supset$ & $\supset$ & $\supset$ & $\infty$ & $\supset$ & $\supset$ & $D$ & د & $\supset$ \\
\hline 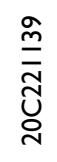 & $\begin{array}{l}\frac{\text { O্ }}{\bar{Z}} \\
\text { U্ }\end{array}$ & $\begin{array}{l}\bar{\Xi} \\
\overline{\bar{N}} \\
\text { ত্ }\end{array}$ & $\frac{\mathcal{Z}}{\bar{Z}}$ & 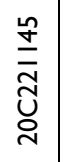 & 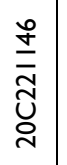 & 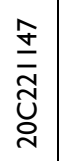 & 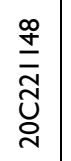 & 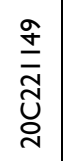 & 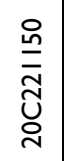 & $\begin{array}{l}\bar{\sigma} \\
\overline{\widetilde{N}} \\
\text { ত্ন }\end{array}$ & 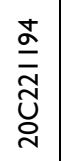 & $\begin{array}{l}\stackrel{2}{\varrho} \\
\overline{\widetilde{Z}} \\
\stackrel{\widetilde{N}}{0}\end{array}$ & 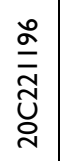 & 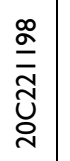 & 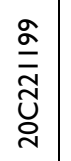 & 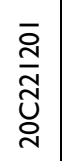 &  &  & $\begin{array}{l}\hat{m} \\
\overline{\bar{N}} \\
\text { ত্ } \\
\end{array}$ & 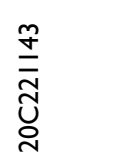 \\
\hline$\ddot{\sim}$ & $\stackrel{\sim}{\Delta}$ & $\stackrel{\mathscr{\sim}}{\sim}$ & $\stackrel{2}{\sim}$ & $\hat{\sim}$ & $\stackrel{\infty}{\sim}$ & సి & ᄋ్ల & $\bar{m}$ & $\tilde{m}$ & $\stackrel{m}{m}$ & ఉ్ & $\stackrel{\stackrel{n}{m}}{n}$ & లి & $\hat{m}$ & $\stackrel{\infty}{m}$ & mे & 우 & $\bar{\gamma}$ & $\mathcal{F}$ & $\stackrel{m}{\forall}$ \\
\hline
\end{tabular}




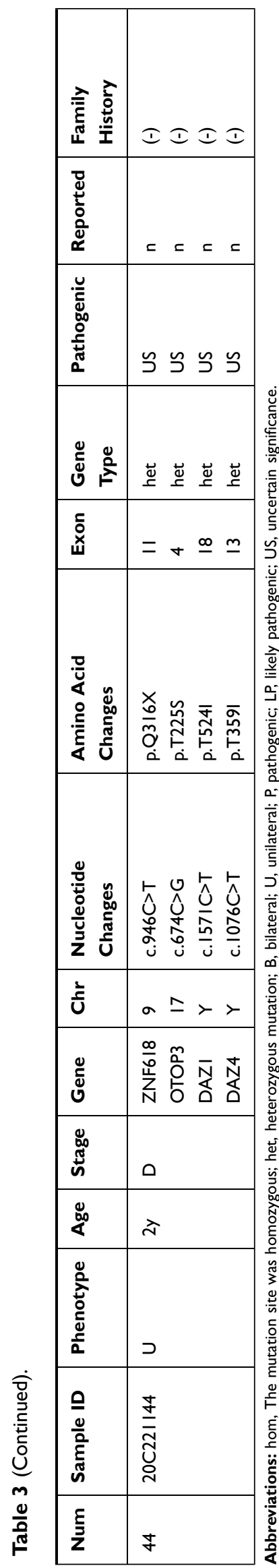

\section{Peripheral Blood Samples}

All 12 peripheral blood specimens were found to have RB1 mutations, with a positivity of $100 \%$. The mutations are summarized in Table 4 . These results indicate that a high detection rate can be achieved using blood testing, the samples for which are easily available in clinic.

\section{Unreported Mutations FFPE Tissue Samples}

There were four cases of unreported RB1 mutations in the FFPE tissues, three of were pathogenic mutations and one of which was a potentially pathogenic mutation, as shown in Table 3.

\section{Peripheral Blood Samples}

Three types of potentially pathogenic mutations that had not been reported before were detected in the peripheral blood samples, as shown in Table 4.

\section{Analysis of the Sequencing Results}

RB1 mutations were present in 40 of the patients from whom FFPE tissue samples were taken, of which 33 were pathogenic mutations, including 20 single-nucleotide variant (SNV)/stopgain, eight deletion/frameshift mutations, three insertion/shift mutations, and two splicing/splicing mutations. These pathogenic mutations mainly manifested as stopgain and frameshift mutations. A further four cases were potentially pathogenic mutations, including three splicing/splicing and one insertion/non-frameshift, while three cases were mutations with uncertain significance, all of which were SNV/non-synonymous. After gene capture in combination with Sanger sequencing, exon sequencing identified the pathogenic mutations in the FFPE tissue samples as c.1423A $>$ T p.K475X on exon 16, c.1103dupT p.I369Nfs*8 on exon 11, and c.618dupA p.Q207Tfs*5 on exon 7. The potentially pathogenic mutation identified was c.1980_1981insCGG p.L660delinsLR on exon 20. Unreported mutations in the RB1 gene were found in four patients (see Table 3). Three of these cases were pathogenic mutations and one were potentially pathogenic mutations.

All 12 peripheral blood samples exhibited RB1 mutations, of which three were pathogenic mutations, including one deletion/frameshift and two SNV/stopgain. A further six of the peripheral blood samples exhibited potentially pathogenic mutations, while three had mutations of uncertain significance, all of which were SNV/non-synonymous. The types of mutation were diverse and scattered across multiple exonic sequences of RB1 (see Figure 1). Testing of the DNA 
Table 4 Summary of Blood-DNA Genetic Test Results

\begin{tabular}{|c|c|c|c|c|c|c|c|c|c|c|c|}
\hline Number & $\begin{array}{l}\text { Sample } \\
\text { ID }\end{array}$ & Phenotype & Age & Stage & Nucleotide & Amino_Acid & Exon & $\begin{array}{l}\text { Gene } \\
\text { Type }\end{array}$ & Pathogenic & Reported & $\begin{array}{l}\text { Family } \\
\text { History }\end{array}$ \\
\hline 1 & $19 C 078277$ & B & ly & $\mathrm{E}$ & c. $2252 C>A$ & p.S75IY & 22 & het & LP & $\mathrm{n}$ & $(-)$ \\
\hline 2 & $19 C 078278$ & $U$ & ly & D & c. $2213 \mathrm{C}>\mathrm{A}$ & p.T738K & 22 & het & LP & $\mathrm{n}$ & $(-)$ \\
\hline 3 & $19 C 078279$ & $U$ & $8 m$ & D & c. $2260 \mathrm{G}>\mathrm{T}$ & p.V754L & 22 & het & LP & $\mathrm{n}$ & $(-)$ \\
\hline 4 & $19 C 078280$ & U & ly & D & c.2048delT & p.F684Sfs*12 & 20 & het & $P$ & $y^{31}$ & $(-)$ \\
\hline 5 & |9C07828| & $U$ & $10 \mathrm{~m}$ & $\mathrm{E}$ & c. $2217 \mathrm{C}>\mathrm{A}$ & p.F739L & 22 & het & US & $\mathrm{n}$ & $(-)$ \\
\hline 6 & $19 C 078282$ & $U$ & ly & $\mathrm{E}$ & c. $2217 \mathrm{C}>\mathrm{A}$ & p.F739L & 22 & het & US & $\mathrm{n}$ & $(-)$ \\
\hline 7 & $19 C 078283$ & $U$ & $4 y$ & D & c. $2252 \mathrm{C}>\mathrm{A}$ & p.S75IY & 22 & het & LP & $\mathrm{n}$ & $(-)$ \\
\hline 8 & $19 C 078284$ & $U$ & $8 m$ & $\mathrm{E}$ & c. $2260 \mathrm{G}>\mathrm{T}$ & p.V754L & 22 & het & LP & $\mathrm{n}$ & $(-)$ \\
\hline 9 & $19 C 078285$ & B & ly & $\mathrm{E}$ & c. $2265 \mathrm{C}>\mathrm{A}$ & p.F755L & 22 & het & US & $\mathrm{n}$ & $(-)$ \\
\hline 10 & $19 C 078286$ & $U$ & $2 y$ & $\mathrm{E}$ & c. $2252 \mathrm{C}>\mathrm{A}$ & p.S75IY & 22 & het & LP & $\mathrm{n}$ & $(-)$ \\
\hline 11 & $20 C 221197$ & $U$ & ly & E & c. $958 \mathrm{C}>\mathrm{T}$ & p.R320X & 10 & het & $P$ & $y^{17}$ & $(-)$ \\
\hline 12 & $21 C 269444$ & $U$ & ly & D & c. $958 \mathrm{C}>\mathrm{T}$ & p.R320X & 10 & het & $P$ & $y^{17}$ & $(-)$ \\
\hline
\end{tabular}

Abbreviations: het, heterozygous mutation; B, bilateral; U, unilateral; P, pathogenic; LP, likely pathogenic; US, uncertain significance.

from the blood samples identified three potentially pathogenic mutations that had not been previously reported (see Table 4). These mutations included c.2252C $>$ A p.S751Y (three cases), c.2260G $>\mathrm{T}$ p.V754L (two cases), and c.2213C $>$ A p.T738K (one case) on exon 22. By searching the dbSNP and 1000 Genomes Project database to exclude the possibility of SNP, it was concluded that these mutations were pathogenic mutations for RB. No mutations were detected in the patients' parents, suggesting that all these RB1 mutations were de novo mutations.

In the present study, 22 variants (two bilateral and 20 unilateral) were located in the regions encoding two conserved domains of the $\mathrm{pRb}-\mathrm{A}$ (residue 393-572) and B (residue 646-772) — of which nine were stopgain, five were frameshift, four were splicing, three were nonsynonymous, and one was non-frameshift (see Figure 1). Of these, $72.7 \%(16 / 22)$ of cases were pathogenic, with two of these cases being de novo mutations, and $27.3 \%$ (6/ 22) of cases were potentially pathogenic, with four of these cases being de novo mutations.

\section{Discussion}

The RB1 gene, which has an anticancer effect as a tumorsuppressor gene, is the first human anticancer gene to have

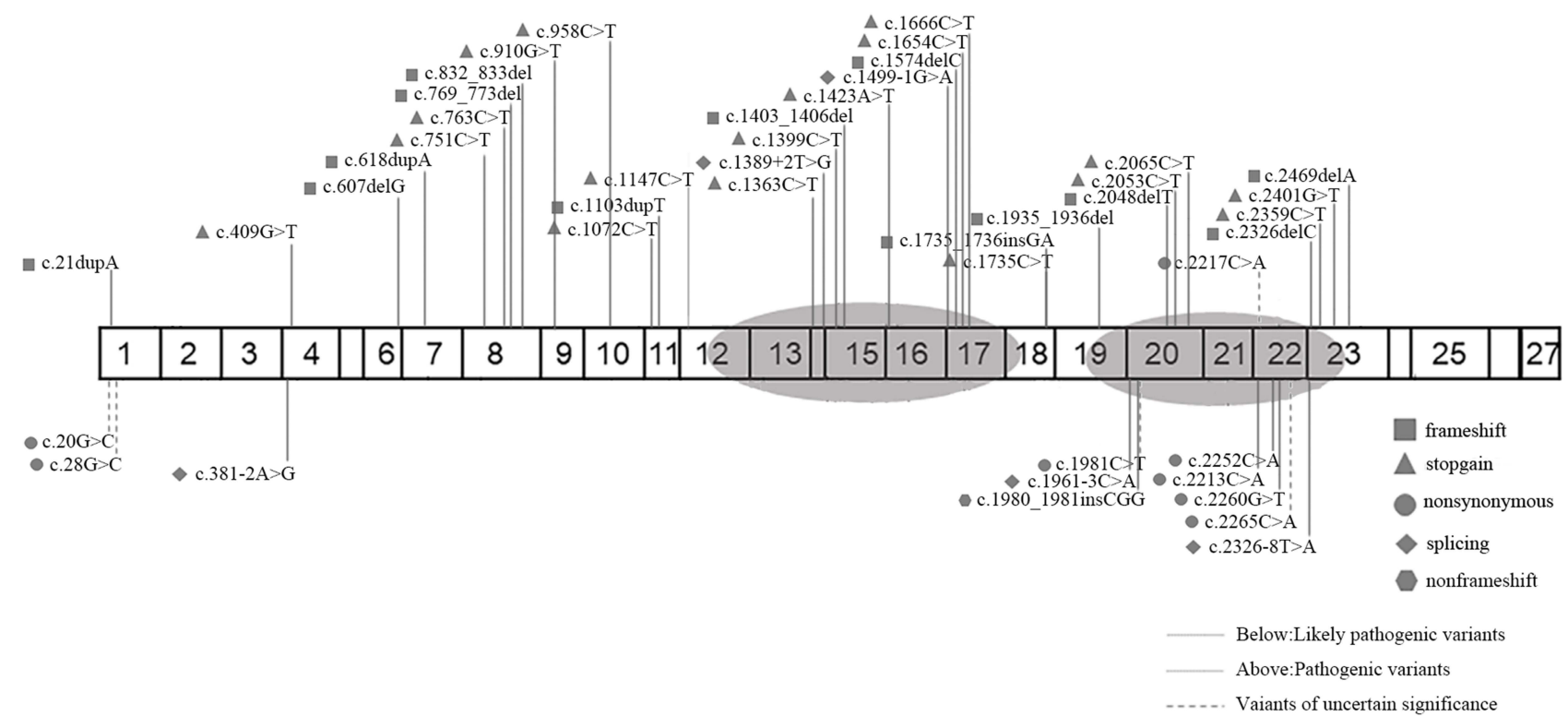

Figure I The distribution of mutation types in the 27 exons and splice sites of the gene. The numbers in boxes refer to the exons, and the two grey oval regions represent functional domains A and B. The five symbols denote five mutation types; the full lines above and below the gene schematic refer to pathogenic and likely pathogenic mutations; dotted lines represent uncertain significance. 
been isolated. Deletion or inactivation of the RB1 gene is an important mechanism for the occurrence of RB, which may occur as a result of the simultaneous deletion, mutation, or inactivation of one pair of RB alleles. The analysis of RB1 mutations and the identification of the genetic type of $\mathrm{RB}$ are important for the determination of a therapeutic plan and follow-up for patients with RB, as well as for the prevention of recurrence for their family members.

The RB1 gene is large, and its mutations are highly heterogeneous and can manifest not only at the chromosomal level, such as variations in chromosome structure and number, but also at the molecular level, such as changes in nucleic acids (substitutions, deletions, insertions, and duplication mutations), deletion of exons, loss of heterozygosity, and transcriptional inactivation due to hypermethylation of the $\mathrm{CpG}$ island in the promoter of the RB1 gene. ${ }^{32}$

The number of new cases of RB is increasing yearly, so it is necessary to establish a rapid and effective RB1 genetesting platform so that the results of gene mutation can be identified quickly for early diagnosis and treatment. Gene capture allows high-throughput sequencing of multiple target regions of pathogenic genes, reducing sequencing costs, shortening the detection duration, and providing high detection rates. ${ }^{33}$ The technique also allows for the selection of different sequencing throughputs, with higher detection rates and better targeting at $1000 \times$ sequencing depth. The clinical selection of different throughputs for pediatric patients with different degrees of RB phenotype may improve the detection rate.

Among the 44 patients in the present study with positive FFPE results, 40 had RB1 gene mutations. Four of the cases were other types of mutations that had no correlation with the RB1 gene: the most common unrelated mutated genes were testis-specific protein Y-linked (TSPY [chr Y]), zinc finger protein 207 (ZNF207 [chr 17]), zinc finger protein 618 (ZNF618 [chr 9)]), otopetrin 3 (OTOP3 [chr 17]), and deleted in azoospermia (DAZ [chr Y]) (see Table 3). The MutRatios (number of mutant bases sequencing/total number of sequencing) of these genes were between 0.01 and 0.03 , with a low value and no need reference. Their presence may have been caused by the DNA decomposition and fragmentation of FFPE tissue affecting the test results.

The results of the present study confirm the diversity of the types of mutation of the RB1 gene. The reported and unreported mutation types identified in the results included deletion, insertion, SNV, and splicing mutation, and the loci were distributed in multiple exons, with exons 22,8 , 23, 17, 20, 10, and 14 being the most common ones. Previous literature has reported that RB1 mutations are mostly nonsense mutations, insertion or deletion mutations, missense mutations, large-fragment deletions, and other mutation types. ${ }^{34,35}$ Valverde et $\mathrm{al}^{36}$ conducted a meta-analysis of 932 reported RB1 mutations in Europe and the USA, identifying three characteristics of the RB1 mutation profile: (1) the mutations are most frequently nonsense mutations, with the incidence of shift mutations, splice-site mutations, and missense mutations caused by small deletions/insertions decreasing in descending order. $\mathrm{pRB}$ is frequently inactivated by nonsense and deletion mutations; (2) although RB1 mutations are scattered throughout the gene sequence, there are 16 hotspot mutations with high recurrence frequency and seven exons with high mutation frequency (exons 9, 10, 14, 17, 18, 20, and 23); (3) the prevalence of RB1 nonsense mutations and splice mutations differs significantly between patients of different races, countries, and regions.

In a study by Sagi et al in Israel ${ }^{37}$ and another by Zou et $\mathrm{al}^{38}$ in Shanghai, China, the most common type of RB1 mutation found was nonsense mutation, followed by splice and shift mutations, with no statistically significant differences between different types of mutation in terms of average age at diagnosis and the side affected by RB. The results of the present study identified the same highfrequency mutation types and mutation loci.

Patients with bilateral RB carry germ-cell mutations, and the literature indicates that $25 \%$ of patients have a family history of the mutation - ie, the gene is inherited from parents with $\mathrm{RB}$ - while the other $75 \%$ are scattered cases and the result of germ-cell mutations during the early embryonic stage. ${ }^{39}$ In the present study, germ-cell mutations were found in four patients with bilateral $R B$, none of whose parents were found to have RBl mutations, which is consistent with the scattered genotype.

In the present study, one patient was selected for simultaneous sequencing of FFPE tissue and peripheral blood DNA, the results of which are shown in Table 5. The sequencing results showed that the FFPE tissue sample exhibited more pathogenic mutation loci than the blood sample, but the MutRatio of the two different mutations was 0.01 , which could be ignored. Thus, the common mutation was c.958C $>$ T p.R320X, indicating concordance between the FFPE tissue and blood sample. This suggests that, when the genetic testing of children is performed clinically, venous blood has the same detection rate as 
Table 5 FFPE-Blood-DNA RBI Gene Test Results

\begin{tabular}{|l|l|l|l|l|l|}
\hline Tissue & cDNA & Protein & Exon & Pathogenic & MutRatio \\
\hline FFPE & c.958C>T & p.R320X & 10 & $P$ & 0.52 \\
& c.1508delC & p.S503Ffs*16 & 17 & $P$ & 0.01 \\
& c.1555_I556insG & p.N519Rfs*4 & 17 & P & 0.01 \\
\hline Blood & c.958C>T & p.R320X & 10 & P & 0.34 \\
\hline
\end{tabular}

Note: MutRatio=number of mutant bases sequencing /total number of sequencing. Abbreviation: $\mathrm{P}$, pathogenic.

pathological tissue sections. However, it is easier to obtain venous blood samples and implement the testing in a clinical setting.

$\mathrm{RB}$ is the most common intraocular malignant tumor in infants and children, and many cases in China have severely affected vision or blindness at the time of diagnosis. The condition can even be life threatening to children. Early detection and early treatment can save a patient's vision, preserve the eye structure, and prolong the patient's life. There is currently an urgent need to improve the coverage of genetic testing and counseling for patients with RB in China, and RB1 genetic testing can help achieve individualized, comprehensive treatment and genetic guidance for patients with RB. The application of genetic testing in the clinical diagnosis and treatment of $\mathrm{RB}$ also needs to be promoted. The China Retinoblastoma Diagnosis and Treatment Guidelines (2019) state that the first signs of RB are leukocoria, strabismus, and other less common signs. After appearance of these signs, patients should be examined by an ophthalmologist and, in case of a possible tumor, should be referred to a specialist for urgent implementation of treatment. After initiation of treatment, genetic analysis should be performed: probands should be tested for the RB1 gene, and patients with chromosome 13q14 deletion should be examined at an ophthalmology department within 48-72 hours; different control measures should be conducted for individuals with known family mutations in the RB1 gene. ${ }^{14}$

Once $\mathrm{RB}$ is diagnosed, its treatment depends on its laterality and the stage of the tumor. Genetic diagnosis is important in predicting the risk of the disease in the siblings or children of patients with $\mathrm{RB}$, thereby reducing the incidence of children being born with $\mathrm{RB}$, improving the overall quality of the population, reducing the psychological and financial burden on families of patients with RB, and saving medical resources. ${ }^{40}$ In the present study, it was found that patients with unilateral RB might carry germline mutations that can be passed on to their offspring, so the application of genetic diagnosis should also be emphasized in patients with unilateral RB, genetic counseling should be actively provided to patients and their families, and early diagnosis and treatment should be provided to high-risk families.

In this study, there were 52 patients with unilateral RB and four with bilateral RB. No standardized selection was made in quantity. Due to the influence of the COVID-19 pandemic, there was a significant decrease in the number of children seeking medical treatment. Most of the patients seeking medical treatment had unilateral RB, and most of those undergoing eye extraction also had unilateral RB. A further study needs to select more patients with bilateral RB, which will be advantageous to the analysis of germcell mutation types. In the present study, the patients with unilateral RB all tested positive for RB1 mutation, which may be due to the severe condition of the children who visited the hospital, all of whom were grade $\mathrm{D}$ or E. Therefore, the number of blood samples collected should be increased in the next study in order to improve the objectivity of the results.

\section{Conclusion}

Gene capture is a low-cost and efficient method for the gene sequencing of RB. A total of seven de novo mutations were identified through mutation testing of the pathogenic gene RB1 in 56 pediatric patients with RB. This complemented the mutation spectrum of the RB1 gene and helped to improve the molecular diagnosis of RB, thereby providing a basis for genetic counseling and prediction of the clinical phenotype, as well as for the genetic testing of the offspring of patients with RB.

\section{Data Sharing Statement}

The datasets used and/or analysed during the current study available from the corresponding author (weiwb1023@163. com) on reasonable request. 


\section{Ethics Approval and Consent to Participate}

This study was conducted in accordance with the declaration of Helsinki.This study was conducted with approval from the Ethics Committee of Beijing Tongren Eye Center. Ethical Approval number TRECKY2018-056.A written informed consent was obtained from legal guardians of all participants.

\section{Funding}

Supported by The Capital Health Research and Development of Special (2020-1-2052); Science \& Technology Project of Beijing Municipal Science \& Technology Commission (Z201100005520045, Z181100 001818003); the Beijing Municipal Administration of Hospitals' Ascent Plan (DFL20150201).

\section{Disclosure}

The authors report no conflicts of interest in this work.

\section{References}

1. Knudson AG Jr. Mutation and cancer: statisticalstudy of retinoblastoma. Proc Natl Acad Sci USA. 1971;68(4):820-823. doi:10.1073/pnas.68.4.820

2. Pandey AN. Retinoblastoma: anoverview. Saudi J Ophthalmol. 2014;28(4):310-315. doi:10.1016/j.sjopt.2013.11.001

3. Kivela T. The epidemiological challenge of the most frequent eye cancer: retinoblastoma, an issue of birth and death. Br J Ophthalmol. 2009;93(9):1129-1131. doi:10.1136/bjo.2008.150292

4. Abramson DH, Frank CM. Second nonocular tumors in survivors of bilateral retinoblastoma: apossible age effect on radiation-related risk. Ophthalmology. 1998;105(4):573-580. doi:10.1016/S0161-6420(98) 94006-4

5. Wu ZY. Orbital Pathology of Modern Eye Tumors. Beijing: People's Military Medical Press; 2002:251-272

6. Rehurek J. History of retinoblastoma. Cesk Slov Oftalmol. 1997;53 (6):351-355.

7. Naumova A, Sapienza C. The genetica of retinoblastoma, revisited. Am J Hum Genet. 1994;54(2):264-273.

8. de Jong MC, Kors WA, de Graaf P, Castelijns JA, Kivelä T, Moll AC. Trilateral retinoblastoma: a systematic review and meta-analysis. Lancet Oncol. 2014;15(10):1157-1167. doi:10.1016/S1470-2045(14) 70336-5

9. Temming P, Arendt M, Viehmann A, et al. How eye-preserving therapy affects long-term overall survival in heritable retinoblastoma survivors. J Clin Oncol. 2016;34(26):3183-3188. doi:10.1200/ JCO.2015.65.4012

10. Alberti WE, Sagerman RH. Diagnosis and management of retinoblastoma. Cancer Control. 2004;11(5):317-327. doi:10.1177/ 107327480401100506

11. Friend SH, Bernards R, Rogelj S, et al. A human DNA segment with properties of the gene that predisposes to retinoblastoma and osteosarcoma. Nature. 1986;323(6089):643-646. doi:10.1038/323643a0

12. Hensey CE, Hong F, Durfee T, Qian YW, Lee EY, Lee WH. Identification of discrete structural domains in the retinoblastoma protein. N-terminal domain is required for its oligomerization. J Biol Chem. 1994;269(2):1380-1387. PMID:8288605. doi:10.1016/ S0021-9258(17)42269-1
13. Dimaras H, Khetan V, Halliday W, et al. Loss of RB1 induces non-proliferative retinoma: increasinggenomic instability correlates with progression to retinoblastoma. Hum Mol Genet. 2008;17 (10):1363-1372. PMID:18211953. doi:10.1093/hmg/ddn024

14. Xu X, Zhao MW. Chinese guidelines for the diagnosis and treatment of retinoblastoma (2019). Chin J Ophthalmol. 2019;55(10):726-738. doi: $10.1159 / 000509424$

15. Woods MO, Williams $\mathrm{P}$, Careen A, et al. A new variant database for mismatch repair genes associated with lynch syndrome. Hum Mutat. 2007;28(7):669-673. doi:10.1002/humu.20502

16. Lohmann DR, Brandt B, Höpping W, Passarge E, Horsthemke B. Spectrum of small length germline mutations in the RB1 gene. Hum Mol Genet. 1994;3(12):2187-2193. doi:10.1093/hmg/3.12.2187

17. Cowell JK, Smith T, Bia B. Frequent constitutional C to T mutations in CGA-arginine codons in the RB1 gene produce premature stop codons in patients with bilateral (hereditary) retinoblastoma. Eur J Hum Genet. 1994;2(4):281-290. doi:10.1159/000472372

18. Blanquet V, Turleau C, Gross-Morand MS, Sénamaud-Beaufort C, Doz F, Besmond C. Spectrum of germline mutations in the RB1 gene: a study of 232 patients with hereditary and non hereditary retinoblastoma. Hum Mol Genet. 1995;4(3):383-388. doi:10.1093/hmg/4.3.383

19. Houdayer C, Dehainault C, Mattler C, et al. Evaluation of in silico splice tools for decision-making in molecular diagnosis. Hum Mutat. 2008;29(7):975-982. doi:10.1002/humu.20765

20. Nájera C, Sánchez F, Mateu E, Prieto F, Beneyto M. Diagnóstico temprano del retinoblastoma: importancia de la búsqueda de mutaciones en el gen RB1 [Early diagnosis of retinoblastoma: usefulness of searching for RB1 gene mutations]. Med Clin (Barc). 2001;116 (10):365-372. doi:10.1016/S0025-7753(01)71832-5

21. Szijan I, Lohmann DR, Parma DL, Brandt B, Horsthemke B. Identification of RB1 germline mutations in Argentinian families with sporadic bilateral retinoblastoma. J Med Genet. 1995;32 (6):475-479. doi:10.1136/jmg.32.6.475

22. Yandell DW, Campbell TA, Dayton SH, et al. Oncogenic point mutations in the human retinoblastoma gene: their application to genetic counseling. $N$ Engl J Med. 1989;321(25):1689-1695. doi:10.1056/NEJM198912213212501

23. Price EA, Price K, Kolkiewicz K, et al. Spectrum of RB1 mutations identified in 403 retinoblastoma patients. J Med Genet. 2014;51 (3):208-214. doi:10.1136/jmedgenet-2013-101821

24. Lohmann DR, Brandt B, Höpping W, Passarge E, Horsthemke B. The spectrum of RB1 germ-line mutations in hereditary retinoblastoma. Am J Hum Genet. 1996;58(5):940-949.

25. Aggarwala V, Ganguly A, Voight BF. De novo mutational profile in RB1 clarified using a mutation rate modeling algorithm. $B M C$ Genomics. 2017;18(1):155. doi:10.1186/s12864-017-3522-z

26. Richter S, Vandezande K, Chen N, et al. Sensitive and efficient detection of RB1 gene mutations enhances care for families with retinoblastoma. Am J Hum Genet. 2003;72(2):253-269. doi:10. $1086 / 345651$

27. Blanquet V, Gross MS, Turleau C, Sénamaud-Beaufort C, Doz F, Besmond $\mathrm{C}$. Three novel germline mutations in exons 8 and 18 of the retinoblastoma gene. Hum Mol Genet. 1994;3(7):1185-1186. doi:10. $1093 / \mathrm{hmg} / 3.7 .1185$

28. Zhang Q, Minoda K, Zeng R, et al. Exon-by-exon screening for RB germline mutations using Heteroduplex-SSCP analysis. Yan Ke Xue Bao. 1997;13(1):5-11.

29. Onadim Z, Hogg A, Baird PN, Cowell JK. Oncogenic point mutations in exon 20 of the RB1 gene in families showing incomplete penetrance and mild expression of the retinoblastoma phenotype. Proc Natl Acad Sci U S A. 1992;89(13):6177-6181. doi:10.1073/ pnas.89.13.6177

30. Tomar S, Sethi R, Sundar G, Quah TC, Quah BL, Lai PS. Mutation spectrum of RB1 mutations in retinoblastoma cases from Singapore with implications for genetic management and counselling. PLoS One. 2017;12(6):e0178776. doi:10.1371/journal.pone.0178776 
31. Lohmann D, Horsthemke B, Gillessen-Kaesbach G, Stefani FH, Höfler H. Detection of small RB1 gene deletions in retinoblastoma by multiplex PCR and high-resolution gel electrophoresis. Hum Genet. 1992;89(1):49-53. doi:10.1007/BF00207041

32. de Andrade AF, da Hora Barbosa R, Vargas FR, et al. A molecular study of first and second RBl mutational hits in retinoblastoma patients. Cancer Genet Cytogenet. 2006;167(1):43-46. doi:10.1016/ j.cancergencyto.2005.08.017

33. Ng SB, Turner EH, Robertson PD, et al. Targeted capture and massively parallel sequencing of 12 human exomes. Nature. 2009;461(7261):272-276. doi:10.1038/nature08250

34. Ayari-Jeridi H, Moran K, Chebbi A, et al. Mutation spectrum of RB1 gene in unilateral retinoblastoma cases from Tunisia and correlations with clinical features. PLoS One. 2015;10(1):e0116615. doi:10.1371/ journal.pone.0116615

35. Abidi O, Knari S, Sefri H, et al. Mutational analysis of the RB1 gene in Moroccan patients with retinoblastoma. Mol Vis. 2011;17:35413547
36. Valverde JR, Alonso J, Palacios I, Pestaña A. RB1 gene mutation up-date, a meta-analysis based on 932 reported mutations available in a searchable database. BMC Genet. 2005;6(1):53. doi:10.1186/14712156-6-53

37. Sagi M, Frenkel A, Eilat A, et al. Genetic screening in patients with Retinoblastoma in Israel. Fam Cancer. 2015;14(3):471-480. PMID:25754945. doi:10.1007/s10689-015-9794-Z

38. Zou Y, Li J, Hua P, Liang T, Ji X, Zhao P. Spectrum of germline mutations in RB1 in Chinese patients with retinoblastoma: application of targeted next-generation sequencing. Mol Vis. 2021; 27:1-16.

39. Rodriguez-Galindo C, Orbach DB, VanderVeen D. Retinoblastoma. Pediatr Clin North Am. 2015;62(1):201-223. doi:10.1016/j.pcl.20 14.09.014

40. Chen CZ, Yu XX. Attention should be paid to the genetic diagnosis of retinoblastoma. Chin J Exp Opthalmol. 2013;31(7): $617-620$.
Risk Management and Healthcare Policy

\section{Publish your work in this journal}

Risk Management and Healthcare Policy is an international, peerreviewed, open access journal focusing on all aspects of public health, policy, and preventative measures to promote good health and improve morbidity and mortality in the population. The journal welcomes submitted papers covering original research, basic science, clinical \& epidemiological studies, reviews and evaluations,

\section{Dovepress}

guidelines, expert opinion and commentary, case reports and extended reports. The manuscript management system is completely online and includes a very quick and fair peer-review system, which is all easy to use. Visit http://www.dovepress.com/testimonials.php to read real quotes from published authors. 